\title{
A Pharmacokinetic Study of Intramuscular Administration of Bulleyaconitine A in Healthy Volunteers
}

\author{
Wei-Yu Weng, ${ }^{a}$ Hui-Nan Xu, ${ }^{* a}$ Jian-Ming Huang, ${ }^{b}$ Guo-Quan WAng, ${ }^{c}$ Teng Shen, ${ }^{a}$ and \\ Jian-Fang ZHANG $^{a}$ \\ ${ }^{a}$ Department of Pharmaceutics, School of Pharmacy, Fudan University; 138 Yixueyuan Road, Shanghai 200032, People's \\ Republic of China: ${ }^{b}$ Department of Pharmaceutical analysis, School of Pharmacy, Fudan University; 138 Yixueyuan \\ Road, Shanghai 200032, People's Republic of China; and ${ }^{c}$ Center Laboratory, Shanghai Municipal Center for Disease \\ Control \& Prevention; 1380 Zhongshanxi Road, Shanghai 200336, People's Republic of China. \\ Received November 8, 2004; accepted January 6, 2005
}

The pharmacokinetics of bulleyaconitine A (BLA) after a single dose of $0.2 \mathrm{mg}$ intramuscular injection was evaluated in healthy volunteers. Physical exam, vital signs, clinical laboratory tests and electrocardiogram measurements were monitored to assess the safety and tolerance of the drug. The plasma levels of BLA in serial samples, collected over $15 \mathrm{~h}$, were measured by a validated high-performance liquid chromatography (HPLC)-electrospray ionization tandem mass spectrometry (MS-MS) method. It was demonstrated that BLA was absorbed rapidly after intramuscular injection. The pharmacokinetic parameters were as follows: the $t_{\max }$ value was $0.90 \pm 0.68 \mathrm{~h}$, the $C_{\max }$ value was $1.13 \pm 0.76 \mathrm{ng} / \mathrm{ml}$, the $A U C_{0 \rightarrow}$ was $5.16 \pm 2.05 \mathrm{ng} \cdot \mathrm{h} / \mathrm{ml}$, and $t_{1 / 2}$ was found to be $4.88 \pm 0.97 \mathrm{~h}$. No subject showed any drug-related clinically significant changes on physical examination, vital signs or laboratory tests. Eight of ten subjects reported a distinct feeling of pain at the site of injection starting approximately at the time of their peak plasma concentration and lasting for $2-6 \mathrm{~h}$. The pain was tolerable, and no subject required additional treatment.

Key words bulleyaconitine A; injection; pharmacokinetics

Bulleyaconitine A (BLA) (Fig. 1), a diester-diterpene type Aconitum alkaloid extracted from Aconitum longtounense T. L. MING, is a potent analgesic and anti-inflammatory agent, and can be used for the treatment of rheumatoid arthritis, osteoarthritis, periarthritis humeroscapularis, lumbar muscle strain, sprain, etc. ${ }^{1-3)}$ The analgesic effect of BLA might be related to the 5-HT level in the brain, and no physical dependence has been observed. The relative analgesic effect of BLA was found to be as much as 3, 65, and 7000 times as potent as 3-acetylaconitine, morphine and aspirin, respectively. ${ }^{2,4}$ BLA has been approved by SFDA (Sino Food and Drug Administration) as intramuscular injections, tablets and soft gel capsules for clinical use in China since 1980's.

The potential toxicity of the diester-diterpene type Aconitum alkaloids such as BLA is severe. The $\mathrm{LD}_{50}$ of BLA, injected subcutaneously, is $0.92 \mathrm{mg} / \mathrm{kg}$ for mouse and $0.51 \mathrm{mg} / \mathrm{kg}$ for rat, respectively. ${ }^{1)} \mathrm{In}$ animal study, toxicity reactions such as breath depression, hydrocephalus and ventricular arrhythmia have been reported when large dose of BLA was administrated. ${ }^{1,2)}$ Although the major side reactions of BLA injections in clinical application are nausea, numbness, palpitation, pain feeling and anaphylaxis at the site of injection, ${ }^{1,2)}$ and no death case has been reported, it is desirable to understand the pharmacokinetic characteristics of the drug and to monitor the plasma concentration of BLA during clinical use. However, the blood concentration of BLA is ex-

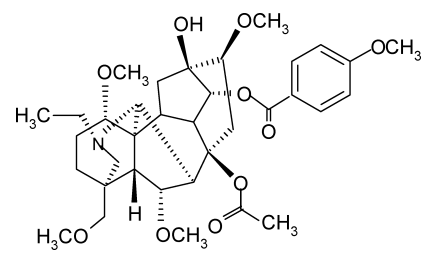

Fig. 1. Chemical Structure of BLA tremely low because of the low dose and rapid metabolism of the drug. No pharmacokinetic data of BLA in human have been reported, likely due to the difficulties encountered with the determination of this compound in biological samples.

In our previous study, we developed a very specific and sensitive method of high-performance liquid chromatography (HPLC)-electrospray ionization tandem mass spectrometry (MS-MS) to detect the concentration of BLA in a human biological matrix. The purpose of this study was to characterize the pharmacokinetics of BLA after a single intramuscular injection in healthy male volunteers.

\section{MATERIALS AND METHODS}

Chemicals BLA standard (the purity is $100.0 \%$ ) was donated by Kunming Pharmaceutical Co. Ltd., and internal standard (I.S.) ketoconazole (99.6\%) was obtained from Nanjing 2nd Pharmaceutical factory. BLA injections $(0.2 \mathrm{mg} / 2 \mathrm{ml}$, Lot20010619) were purchased from Yunnan Pinbian Pharmaceutical Factory. Acetonitrile of HPLC grade was purchased from Merck (Darmstadt Germany). Other reagents used were of analytical grade. Water was deionized, filtered and purified by a Milli-Q Reagent Grade Water System from Millipore (Millipore, Bedford, MA, U.S.A.). Drugfree human heparinized plasma was obtained from Shanghai Blood Center (Shanghai, PR China). Nitrogen (99.999\%) and argon were purchased from Shanghai BOC Gas Industry (Shanghai, PR China).

Instrumentation The mobile phase was delivered by an Alliance 2690 HPLC system (Waters, Milford, MA, U.S.A.) consisted of a quaternary pump, a column heater, an autosampler, a $7725 \mathrm{i}$ injector and a Millennium chromatographic work station. The detection was performed by a Quattro-LC ion trap mass spectrometer (Micromass, Manchester, U.K.) using electrospray ionization (ESI) for ion production and a 
Masslynx work station.

Analytical Methods The plasma concentrations of BLA were determined by a validated high-performance liquid chromatography (HPLC)-electrospray ionization tandem mass spectrometry (MS-MS) method. Briefly, after addition of a suitable internal standard solution, the plasma samples $(1.0 \mathrm{ml})$ were extracted with ether $(5.0 \mathrm{ml})$. The organic phase was recovered, dried and reconstituted for chromatography. The separation was performed on Waters Xterra MS $\mathrm{C}_{18}$ reversed-phase column $(3.5 \mu \mathrm{m}, 2.1 \times 50 \mathrm{~mm})$. The mobile phase composed of a premix of solvent $A(0.1 \%(\mathrm{v} / \mathrm{v})$ acetic acid aqueous solution) and solvent $\mathrm{B}(0.1 \%$ acetic acid acetonitrile solution). The mixture was eluted at $0.3 \mathrm{ml} / \mathrm{min}$ by a gradient method: $40 \% \mathrm{~B}$ from 0 to $1.5 \mathrm{~min}, 40 \% \mathrm{~B}$ to $95 \% \mathrm{~B}$ from 1.5 to $4 \mathrm{~min}$, and $95 \% \mathrm{~B}$ from 4 to $6 \mathrm{~min}$. BLA was determined by multiple reaction monitoring using precursor $\rightarrow$ product ion combinations at $m / z$ 644.6 $\rightarrow 584.3$.

Ethics The study was conducted in full compliance with the principles of Good Clinical Practices. The study protocol, amendments and subject consent forms were reviewed and approved by the Ethics Committee of Base for Drug Clinical Trial of SFDA, Shanghai Zhongshan Hospital, China PR.

Subjects Ten healthy male volunteers (age range $20-25$ years, body weight within $55-75 \mathrm{~kg}$ ) with no clinically significant abnormalities on standard clinical examination were enrolled. The relevant exclusion criteria included: participation in other clinical trials during the previous three months; presence or history of cardiovascular, respiratory, renal, hepatic, gastrointestinal, neurological or other disease; alcohol or drug abuse. The subjects were required to abstain from smoking and ingestion of alcoholic or caffeine-containing beverages. Each participant signed a written informed consent. Two weeks prior to the study, the subjects were screened using their medical history, the results of physical examination, the clinical laboratory investigations, and 12lead electrocardiogram.

Study Design This was an open-label, uncontrolled, single-dose, single-center study in healthy males to determine the pharmacokinetics, safety, and tolerability of BLA after a single dose $0.2 \mathrm{mg}$ intramuscular injection.

Pharmacokinetic Assessments The venous blood samples $(3 \mathrm{ml})$ were collected before the administration and at
$0.17,0.33,0.5,0.75,1,1.5,2,3,4,6,8,10,12$ and $15 \mathrm{~h}$ following dosing. The blood sample was placed in a heparinized tube and centrifuged at $1500 \mathrm{~g}$ for $10 \mathrm{~min}$. The plasma was removed and frozen at $-20^{\circ} \mathrm{C}$ until the analysis.

The following pharmacokinetic parameters were calculated. The peak plasma concentration $\left(C_{\max }\right)$ and the time to peak concentration $\left(t_{\max }\right)$ were derived from the measured values. The area under the plasma concentration-time curve from the time of administration to the time of the last quantifiable plasma concentration $\left(A U C_{0-}\right)$ was calculated by the trapezoidal rule. The extrapolated area under the curve $\left(A U C_{0-\infty}\right)$ was calculated as $A U C_{0-t}=A U C_{0-t}+C_{t} / k_{\mathrm{el}}$, where $C_{t}$ is the last quantifiable concentration, $t$ is the time of $C_{t}$ and $k_{\mathrm{el}}$ is the elimination rate constant determined by log-linear regression analysis on data points on the terminal log-linear phase. The terminal elimination half-life $\left(t_{1 / 2}\right)$ was calculated as $0.693 / k_{\mathrm{el}}$.

Safety Assessment Adverse event data was combined from the information volunteered by the subjects and the reports from the study staff who monitored the subjects. Vital signs (heart rate, blood pressure, and respiratory rate) were monitored prior to administration and at $0.5,1$ and $15 \mathrm{~h}$ after administration, as well as during a follow-up visit. Physical examinations, clinical laboratory tests and 12-lead electrocardiogram measurements were performed at screening, prior to the administration, and at the follow-up visit. The incidence of spontaneously reported adverse events was also recorded.

\section{RESULTS}

Determination of BLA in Plasma Typical chromatograms of BLA in human plasma are shown in Fig. 2. Linearity was established in the concentration range of $0.12-6 \mathrm{ng} / \mathrm{ml}(Y=1.087 X-0.1103, r=0.9990)$. The intraand inter-day relative standard deviation (R.S.D.) of BLA detection ranged from 5.514 to 16.49 and from 6.829 to 19.36 , respectively. The recoveries of the method for BLA ranged from 96.93 to $113.89 \%$. The extraction recovery was from 76.35 to $88.38 \%$.

Pharmacokinetics The pharmacokinetic parameters of BLA injection are summarized in Table 1, and the mean

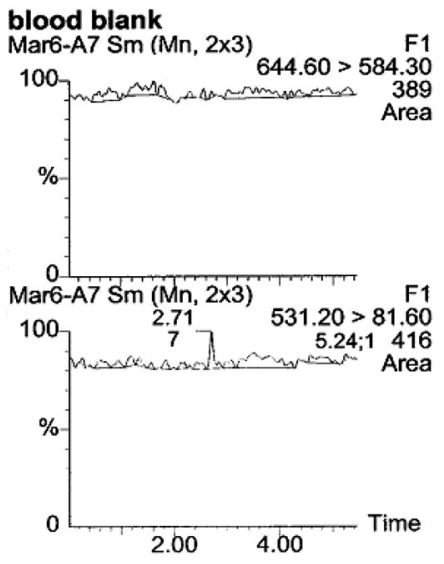

(a)

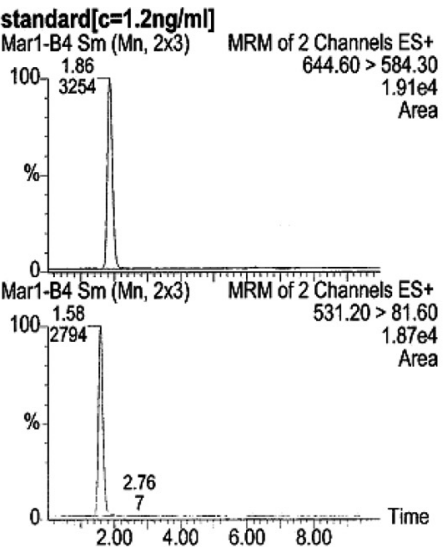

(b)

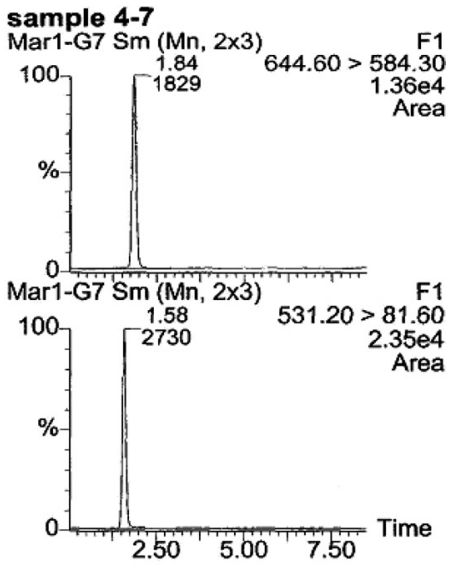

(c)

Fig. 2. Typical Chromatograms of (a) Blank Plasma; (b) Quality Control Sample (1.2 ng/ml); (c) Actual Human Plasma Sample after Single Intramuscular Injection of $0.2 \mathrm{mg}$ BLA ( $2 \mathrm{~h}$ after Administration) 
Table 1. Summary of Pharmacokinetic Parameters

\begin{tabular}{lcccccc}
\hline \hline & $\begin{array}{c}t_{\max } \\
(\mathrm{h})\end{array}$ & $\begin{array}{c}C_{\max } \\
(\mathrm{ng} / \mathrm{ml})\end{array}$ & $\begin{array}{c}A U C_{0-t} \\
(\mathrm{ng} \cdot \mathrm{h} / \mathrm{ml})\end{array}$ & $\begin{array}{c}A U C_{0-\infty} \\
(\mathrm{ng} \cdot \mathrm{h} / \mathrm{ml})\end{array}$ & $\begin{array}{c}K \\
\left(\mathrm{~h}^{-1}\right)\end{array}$ & $\begin{array}{c}t_{1 / 2} \\
(\mathrm{~h})\end{array}$ \\
\hline Mean \pm S.D. & $0.90 \pm 0.68$ & $1.13 \pm 0.76$ & $5.16 \pm 2.05$ & $6.01 \pm 2.40$ & $0.15 \pm 0.03$ & $4.88 \pm 0.97$ \\
Range & $0.33-2.00$ & $0.60-3.05$ & $2.74-9.39$ & $3.18-10.92$ & $0.10-0.19$ & $3.65-6.73$ \\
\hline
\end{tabular}

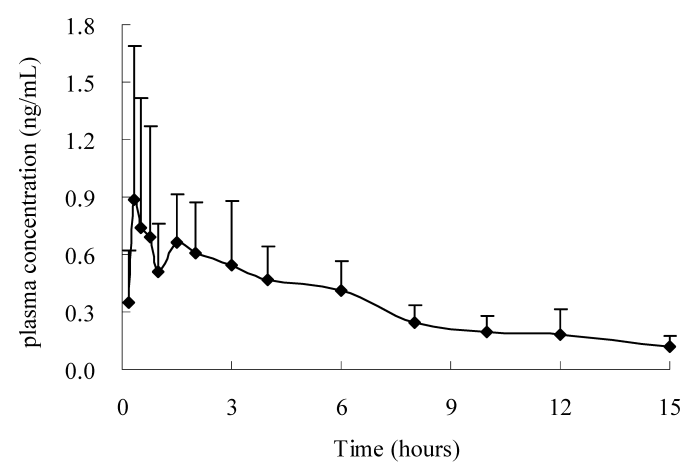

A

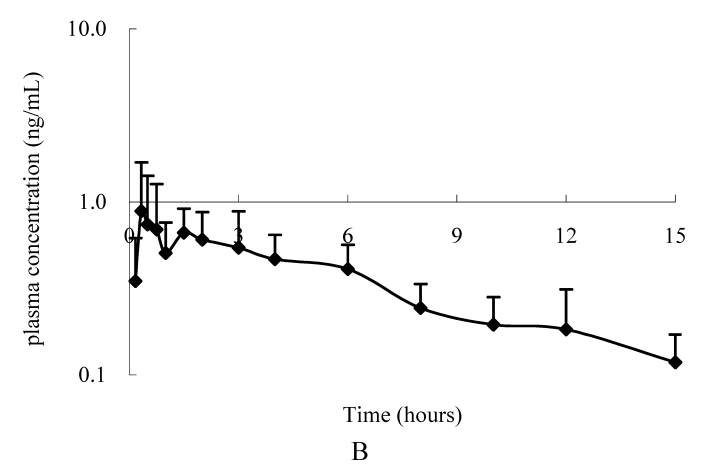

Fig. 3. Mean Plasma Concentrations and Standard Deviations (S.D.) Following Single Intramuscular Administration of BLA $0.2 \mathrm{mg}$

$\mathrm{A}$ is a standard scale for concentration, and B is a log scale for concentration.

plasma concentration-time profile of BLA is illustrated in Fig. 3.

Tolerability All ten healthy male volunteers completed the study. Mean demographic characteristics were: age, $21.4 \pm 1.0$; weight, $62.7 \pm 5.4 \mathrm{~kg}$. No subjects showed any drug-related clinically significant changes in physical exami- nation, vital signs or laboratory tests. Eight subjects reported a distinct feeling of pain at the site of injection starting approximately at the time of their peak plasma concentration and lasting for $2-6 \mathrm{~h}$. The pain was tolerable, and no subject required additional treatment for this adverse event.

\section{DISCUSSION}

As a diester-diterpene type Aconitum alkaloid, BLA undergoes rapid biological metabolism. The results of our previous experiments showed that no prototype drug could be detected in the human plasma after an oral administration of $0.4 \mathrm{mg}$ BLA. The exact biological metabolic kinetics and metabolic products of BLA have not been clarified yet.

In the present paper, we reported, for the first time, the pharmacokinetic data of BLA in human after intramuscular administration. To our best knowledge, it was also the first narration on the clinical pharmacokinetics of Aconitum alkaloids in human. The results indicated that BLA was rapidly absorbed after intramuscular injection. The statistical results of clinical applications of BLA injections showed that after intramuscular injection of $0.2 \mathrm{mg}$, the mean on-set time of the analgetic action was $37.8 \mathrm{~min}$ and that the duration lasted for about $9.3 \mathrm{~h} .{ }^{1)}$ In this period of time, the plasma concentration was in the range of $0.2-0.9 \mathrm{ng} / \mathrm{ml}$, therefore, the minimum effective plasma concentration of BLA was approximately $0.2 \mathrm{ng} / \mathrm{ml}$.

\section{REFERENCES}

1) Sha J. S., Mao H. K., Chin. Pharm. J., 28, 378-379 (1993).

2) Tang X. C., New Drugs Clin. Rem., 5, 120-121 (1986).

3) Zhang Y. C., Yang Q. R., Yin H. E., Liu W. B., Yin G. F., Zhang D. S., Pharmcol. Clin. Chin. Mater. Med., 16, 43-45 (2000).

4) Tang X. C., Liu X. J., Lu W. H., Wang M. D., Li A. L., Act. Pharm. Sinica, 21, 886-891 (1986). 